\title{
Comparing the Effects of Social Robots and Virtual Agents on Exercising Motivation ${ }^{\star}$
}

\author{
Sebastian Schneider and Franz Kummert \\ Applied Informatics, CITEC, Bielefeld University, Bielefeld 33616, Germany \\ $\{$ sebschne,franz\}@techfak. uni-bielefeld.de
}

\begin{abstract}
Preventing diseases of affluence is one of the major challenges for our future society. Researchers introduced robots as a tool to support people on dieting or rehabilitation tasks. However, deploying robots as exercising companions is cost-intensive. Therefore, in our current work, we are investigating how the embodiment of an exercising partner influences the exercising motivation to persist on an abdominal plank exercise. We analyzed and compared data from previous experiments on exercising with robots and virtual agents. The results show that the participants had longer exercising times when paired with a robot companion compared to virtual agents, but not compared to a human partner. However, participants perceived the robots partner as more likable than a human partner. This results have implications for SAR practitioners and are important for the usage of SAR to promote physical activity.
\end{abstract}

Keywords: Socially Assistive Robots · Exercising · Embodiment.

\section{Introduction}

The World Health Organization (WHO) states in their key facts ${ }^{1}$ about physical activity (PA) that insufficient PA is not only one of the leading risk factors for death worldwide but also a key risk factor for Noncommunicable Diseases (NCDs) such as cardiovascular diseases, cancer, and diabetes. However, getting people motivated to increase their $\mathrm{PA}$ is a challenging problem [3]. A variety of factors influence people to start an exercise regimen [3] and having social support is one of the most positively associated factors for adults [16. However, appropriate assistance from peers, coaches or physicians, which could facilitate starting and sticking to a workout, is not available for everybody. It includes finding and scheduling with the associates and often to commute to other places. Hence Socially Assistive Robots (SARs) 6 6 have been introduced as a suitable tool to facilitate motivation because people are likely to anthropomorphize nonbiological artifacts [4] and treat media and technology human-like [12. In our

\footnotetext{
* Supported by grants from the Cluster of Excellence Cognitive Interaction Technology 'CITEC' (EXC 277), Bielefeld University.

${ }^{1}$ All of these facts are on the WHO website:http://www.who.int/en/news-room/ fact-sheets/detail/physical-activity, retrieved 08/14/2018
} 
previous work, we have presented the effects of exercising together with a robot as a partner 14. However, today's technologies allow for changing the representation of the partner easily. Smartphone applications, exercising videos on online platforms or exercising with human partners via internet video calls are possible variations. Those technologies could be used to emulate the feeling of working out together with a partner. Compared to those technology, it raises the question whether Embodied Robot (ER) are necessary in a task where no physical interaction is needed?

Deploying ERs comes with issues regarding their physical ability and their maintenance. Thus, Virtual Agents (VAs) have a substantial advantage over robots, because they are easily deployable, do not have physical limitations, are cheaper and need less care than robots. However, do ERs and VAs elicit the same social and motivational effects? Li [11] tried to answer this question with a recent research survey which shows that in most cases robots are in favor of VAs ${ }^{2}$. However, there are also works that are showing contradicting results 13,9 . While other works investigated the embodiment effects of SAR for rehabilitative tasks [5], we are interested in measuring quantifiable motivational impacts of an embodied SAR during an abdominal exercising task.

Our previous studies showed the motivational effects of working out coactively with a SAR or receiving encouraging feedback from a SAR 14, 15. In the present work, we want to look at whether co-actively exercising robots are enhancing people's motivation to exercise compared to virtual partners.

This work is organized as follows: The next section reviews previous and related works. Section 4 introduces the study design and data acquisition. Section 5 presents the results and section 6 discusses the results. The final section concludes the results and gives an outlook.

\section{Related Work}

Previous works investigated the effects of a robot's embodiment in different tasks. These works include studies on SAR supporting on cognitive tasks [10], which showed that an embodied robot increases a learning gain. A study on authority and personal space in a book moving task showed that people are more willing to obey orders from an embodied agent and give them more personal space [1. However, studies on language learning and teaching showed no differences in terms of learning gains between agent embodiment 9,13 .

A recent meta-review on the benefits of being physically present investigated not only the impact of embodiment but also on co-presence [11]. Their results show that a physically present robot compared to a telepresent robot had stronger effects regarding the participant's response, are more persuasive and increase response time. Compared to virtual agents, co-present robots are more convincing, increase user's attention and response speed, are favored, and users show more positive attitudes towards co-present robots. Regarding the differences between a telepresent and virtual represented robot they did not

\footnotetext{
${ }^{2}$ They distinguished different levels of an agent's presence (i.e., embodied remotelocated vs. virtually represented), but here we are referring to both as VAs
} 
find any differences. The authors conclude that co-present robots have a benefit compared to virtual agents or telepresent robots, but subsequent studies showed contradicting results 9, 13. Thus, it remains an ongoing question what the actual benefits of being physically present are and in which tasks they have an impact.

Regarding the embodiment effects of SAR specially designed for exercising or rehabilitation tasks, there is only one work investigating the impact of robot embodiment in a long-term study [5. They compared a physical robot with its virtual counterpart in a longitudinal study with five 20-minute exercising sessions over a period of two-weeks. Their results provide evidence that users perceive a physically embodied robot as more enjoyable, valuable, helpful and socially attractive compared to the virtual robot. However, these are subjective evaluations from the participants that do not show whether embodied SAR have a quantifiable and observable motivational effect compared to virtual agents.

\section{Hypothesis}

The present research contributes to the ongoing efforts in understanding the effects of embodiment and tries to close the research gap by showing that embodied robots not only are perceived as more sociable, enjoyable and helpful but also increase exercising time. Based on the previous research from 11] and [5] we have the following hypothesis:

A robot companion enhances a human's motivation to persist on an exercise compared to a virtual partner (H1)

To test this hypothesis, we combine the data of the two previously done experiments on abdominal plank exercises with virtual agent partners and robot partners. We analyze the data to investigate whether there is a motivational effect in persisting on the task due to the different partner embodiments.

\section{Planned Data Analysis}

To investigate whether embodied robots show an advantage in terms of exercising motivation compared to VAs, we analyze the data of the previous studies from [14] and [15] and from 7. Feltz et al. 7] conducted a study to compare the motivational effects of exercising with a humanoid virtual partner with a hardly human-like appearance [Hardly Human Partner (HHP) $)$, a nearly human-like appearance (Nearly Human Partner (NHP)) and with a human partner (Human Partner (HP)) (see Figure 2p compared to a condition in which the subject is always exercising alone (Individual Condition (IC)). Their result shows that even though it is a small effect, exercising with a/VA is more motivating than having no partner at all, which we have also investigated for having robot partners. In the previous works we replicated the study of 7 and replaced the $\mathrm{HP}$ and VA with the humanoid robot platform Nao (see Figure 1). However, we included some changes in the study design due to the usage of a robotic agent. We changed the exercises from forearm planks to full planks due to the robot's limited Degree of Freedom (DoF). Additionally, due to the physical limitation in exercising abilities of current humanoid robot platforms, we are interested in whether a robot that 
is exercising co-actively with the user is required. Thus, we previously included a condition where we tested the effects of just having a robot instructor Robot Instructor (RI) .

We showed that a co-actively exercising robot companion Robot Companion (RC) leads to higher motivation to persist the exercises than exercising alone, but not for the robot that is just instructing the user (RI). Adding encouraging feedback leads to greater exercising performance when the robot is instructing (Robot Instructor with Feedback (RIF) but did not lead to higher performance in the companion condition (Robot Companion with Feedback (RCF)). We have attributed this results to ceiling effects due to the difficulty of the exercises ${ }^{3}$. Now, we have the opportunity to compare the persistence data from the studies with robots and compare our results with the results from exercising with virtual companions and humans. For the reader's comprehension, we summarize in the following subsections the planned data analysis and used measurements.

\subsection{Conditions and Experimental Design}

Participants in both studies were assigned to one of nine conditions, which we will explain now.

$H P$ In 7 the Human Partner (HP) was a college-aged partner whose video was prerecorded.

NHP In [7] the Nearly Human Partner (NHP) was the same video as the HP. but with a computerized effect applied to the video.

HHP In [7] the Hardly Human Partner (HHP) were a three-dimensional graphical characters. The character was animated to perform the plank exercises.

$R C$ In [14 the Robot Companion (RC) partner was the humanoid robot platform Nao. Nao's motion were animated using Choregraphe to perform the plank exercises together with the human.

$R I$ In 14 the Robot Instructor (RI) partner was the same humanoid robot platform. However, instead of exercising co-actively with the human, it simply structures the exercise session.

$R C F$ In 15 the Robot Companion with Feedback (RCF) had the same behavior as in the RC. However, the robots also gave encouraging feedback while exercising (see $[15$ for detailed information).

RIF In 15 the Robot Instructor with Feedback (RIF) had the same behavior as in the RI. However, the robot also gave encouraging feedback while exercising.

$I C$ and $I C_{2}$ Are the baseline conditions were participants exercised a second time individually. $[\mathrm{IC}$ is the isolated condition from 7$]$ and $\overline{\mathrm{IC}_{2}}$ is the isolated condition from 14 .

In the robot condition studies, participants $(\mathrm{n}=95)$ were randomly assigned

\footnotetext{
${ }^{3}$ Participants reported in post-study interviews that they would have liked to exercise
} longer but that they had too much pain in the wrist due to the exercises. 


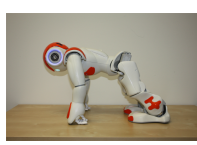

(a) plank
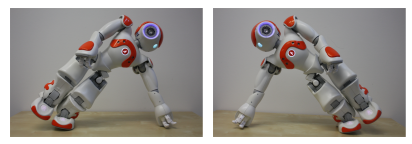

(b) side plank (c) side plank left

right
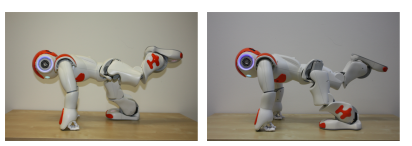

raise left raise right

Fig. 1: The five different abdominal plank exercises used in this study.

to three conditions $\left(\mathrm{IC}_{2}, \mathrm{RC}, \mathrm{RI}, \mathrm{RIF}, \mathrm{RCF}\right)$. Participants were mostly students (51 male, 44 female; mean age $[M]=25.4$ years; standard deviation $[S D]=5.6$ ) from Bielefeld university acquired by flyers distributed on the campus. They received seven Euros as monetary compensation. Three participants from the IC were excluded. One was an outlier already persisting much less during the first part of the session when the participants were exercising by themselves compared to all other participants. Two other persons were excluded because they were doing the exercises wrong. One participant in the RI condition had to be excluded from the survey evaluation because the data were missing. In all other cases, no outliers have been removed.

Feltz et al. 7 randomly assigned participants $(\mathrm{n}=120)$ to four exercise conditions $(\mathrm{IC}, \mathrm{NHP}, \mathrm{HHP}, \mathrm{HP}$ with 30 participants in each condition. Participants were undergraduate students (60 females, 60 males; mean age $[M]=$ 19.41 years; standard deviation $[S D]=1.52$ ) recruited from a large Midwestern university who completed the experiment for course credit.

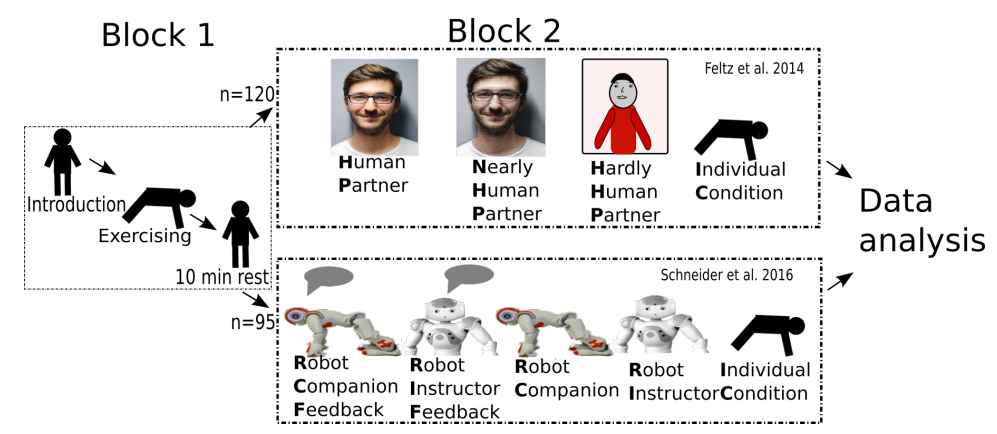

Fig. 2: The study design and conditions that were used to gather the data that are analysed in the present work.

\subsection{Procedure}

The procedure to obtain the data for this analysis is depicted in Figure 2 and was the same for both studies (see 14 or 77 ). Each participant arrived at the lab and was instructed to do five abdominal plank exercises as long as they can (no experimenter was in the same room). Afterward, participants were told that they have to do the five exercises again. They rested for ten minutes and were told their average exercising time during Block 1. Then, the manipulation and the participants were assigned to one of the conditions. In the partner conditions, participants were told that the time of the partner who quits first will 
count and that the partner can exercise on average $40 \%$ longer. This creates an unfavorable comparison which is vital for the Köhler effect and proofed to elicit the highest motivational gain. Each participant exercised with the partner, answered a survey after the experiment, and was debriefed. This is just a brief explanation of the procedure. Due to the paper limitation, we cannot give a full detailed overview of the procedure and would like to ask the reader to look at the referenced papers describing the study procedure in more detail.

\section{$5 \quad$ Results}

In both studies several different measures have been collected. However, we will not use all of them for the evaluation, because we are mainly interested in the perception of the partner and the persistence on the exercises. Thus, the persistence, Godspeed questionnaire [2], and Physical Activity Enjoyment Scale (PAES) 8 were used.

We tested the data for homogeneity of variance using a Levene's Test and for normality using a Shapiro-Wilk test. Since most of the data is not normally distributed, we used non-parametric tests (e.g. Kruskal-Wallis Rank Sum Test (KW-Test) and Wilcoxon rank sum test (WC-Test)).

\subsection{Persistence}

As a primary dependent variable we used the average difference persistence time in seconds between the two blocks (Block 2 - Block 1). This approach controls for individual differences in strength and fitness and shows possible changes in persistence. At first, we compare the exercising times on Block 1 between the two studies from Feltz et al. ( $M=57.7, S D=23.94)$ and Schneider and Kummert $(M=65.45, S D=22.02)$ to assure that the baseline exercising times are equivalent . The exercising time is significantly affected by the studies, $W=3933, p<$ $.01, r=-.19$. This difference is possibly due to the changes in the exercise from forearm planks to full planks, which makes the exercises harder to persist but likely more challenging for the user and thus more interesting to persist them longer. Hence, we decided to adjust the Block 1 measures to compensate for the difference in the exercises. We used the average of participants' exercising time on Block 1 between the two studies as an adjustment value. Participants in study 7. exercised on average 7.78 seconds less in Block 1 than in the studies from 14 and 15. We used this value and added it to the exercising time of Block 1 for the $\mathrm{NHP}$, HHP, and $\mathrm{HP}$ conditions. Figure 3 shows the adjusted results obtained for the average block scores of Block 2 subtracted with the average block score of Block 1. This figure shows the significant difference between the conditions against a base-mean. A 8 (conditions) x 1 (persistence) KW-Test on the adjusted persistence scores showed a significant main effect for the conditions, $H(8)=67.93, p<.001$. Persistence time in the $\mathrm{HP}, \mathrm{RC}, \mathrm{RCF}$ and $\mathrm{RIF}$ conditions is significantly higher against the base-mean. The $\mathrm{IC}, \mathrm{IC}_{2}$ and $\mathrm{HHP}$ conditions are significantly lower than the base-mean. Due to the limitations of the paper, we cannot report the post-hoc analysis after the KW-Test for the 
persistence time comparing the significant differences between the conditions. A KW-Test for difference between the conditions on the perceived PAES showed no significant differences, $H(7)=4.99, p=.66$

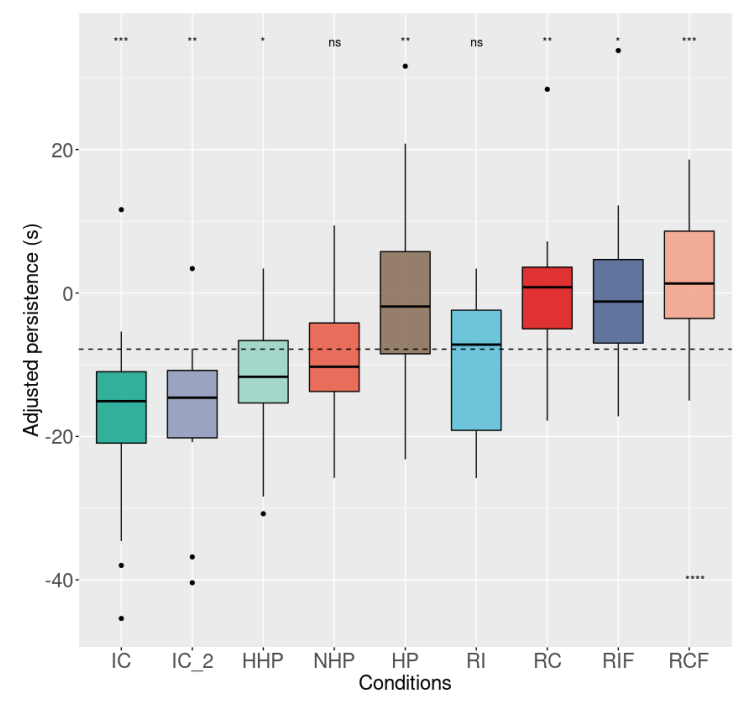

Fig. 3: Average adjusted persistence difference (s) between Block 2 and Block 1 for the different conditions. Comparison against the mean baseline of all conditions.

\subsection{Perception of partner}

We tested the perception of the partner using the four sub-scales of the Godspeed questionnaire (animacy, likability, anthropomorphism and intelligence) using KW-Tests. The scores on the Godspeed questionnaire are shown in Figure 4. We found significant main effects for the perceived animacy of the agents $(H(6)$ $=17.24, p<.01)$, anthropomorphism $(H(6)=21.83, p<.01)$ and likability $(H(6)=30.13, p<.001)$ but not for intelligence $(H(6)=7.03, \mathrm{p}=.31)$. The $\mathrm{HP}$ and $\mathrm{RC}$ are both significantly rated as more animated than then $\mathrm{HHP}$ (we cannot report all the critical differences from the pairwise KW-Test due to the limitation of this paper). The HHP was rated as significantly less anthopomorphic than the $\mathrm{HP}$ and the $\mathrm{RI}$ was rated as significantly less anthropomorphic than the $\mathrm{NHP}$. The $\mathrm{RC}$ and $\mathrm{RCF}$ were perceived as significantly more likable than the $\overline{\mathrm{HP}}$ and $\mathrm{NHP}$. Additionally, the $\mathrm{RCF}$ was rated as significantly more likable than the NHP.

\section{Discussion}

This work aims to fill the knowledge gap on the quantifiable motivational effects of exercising with either a co-located robot or with a virtually represented 


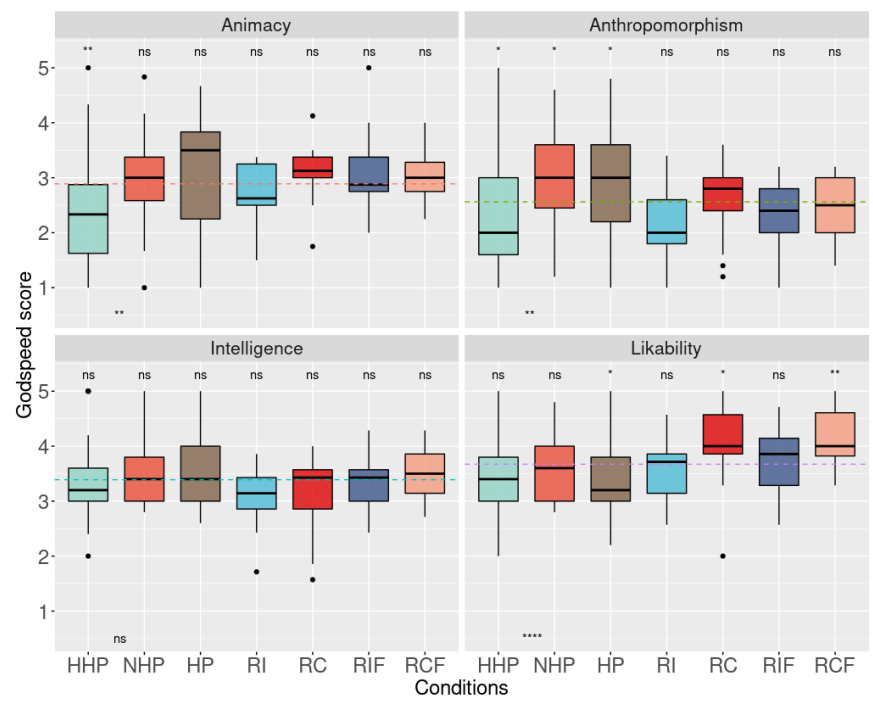

Fig. 4: Scores of the Godspeed questionnaire. Comparison against the mean baseline of all conditions.

agent. We questioned whether the embodiment of an exercising partner increases the motivation to persist during a conjunctive task. The analysis of the available data support our hypothesis H1. Participants in the conditions with a robot companion $(\overline{\mathrm{RC}}$ and $\mathrm{RCF})$ exercised significantly longer than with a virtual partner (NHP and HHP). These results show that the human participants paired with a co-located $[\mathrm{RC}$ are more motivated to exercise longer than with a telepresent or virtual representation of the partner. Moreover, the HP does not elicit a stronger motivational effect compared to a robot. It shows that a robot exercising partner could be at least as motivational as a $\mathrm{HP}$, but more evaluation is needed to assure this. Thus, the studies need to be replicated due to three differences between them.

First, the data have been acquired by two different research groups in different countries. The found effect can be due to cultural differences or subtle difference in the study conduction. Even though we tried to replicate the study as close as possible, we can not guarantee that everything went exactly the same.

Second, we changed the exercises a little bit. Due to the limited DoF of the robot platform, we needed to implement slightly different exercises. The change from forearm plank exercises to full plank exercises results in different exercising times on Block 1. It is likely that the forearm plank exercises were not challenging enough for the participants and thus they instead stopped the exercising due to boredness and not fatigue. This change makes it difficult to compare the data of the two experiments. Our solution was to adjust the exercising time on Block 1 of data from 7 by adding the average difference on this block between the two studies. This approach is reasonable and helps to get an initial view on the motivational effects due to the partner's embodiment, but it still needs to be verified with the same exercises across all conditions. 
Third, the virtual representation of the partner was not the same as the used robot. To be sure that the differences are not due to the representation, the study needs replication with a virtual representation of the same robotic platform. At last, the $\mathrm{HP}$ condition was not a co-located partner as in the robot conditions. This difference in co-location could be an explanation why the persistence in the $\mathrm{HP}$ conditions was not significantly higher than in the robot conditions. A TV displayed the $\mathrm{HP}$, and the experimenters told the participants that the $\mathrm{HP}$ is in a different room connected via a webcam. This difference in the embodiment between the human and robot conditions might also influence the results and shows that future research should target this issue. However, it seems to be almost impossible to conduct such an experiment with a co-located human, since the partner has to be always more capable than the participants to implement the Köhler effect. The need for a more trained exercising partner is a hard requirement that seems to be challenging to fulfill.

The results of the Godspeed questionnaire showd that participants rated the animacy and anthropomorphism of the robot differently between the robot conditions and the $\mathrm{NHP}$ and $\mathrm{HP}$ condition. Furthermore, the results show a difference in likability between the conditions. Participants rated that they liked the robot companion conditions $(\mathrm{RC}$ and $\mathrm{RCF})$ not only more than the $\mathrm{NHP}$ and $\mathrm{HHP}$ condition but also more than in the $\mathrm{HP}$ This difference in perceived likability is an intriguing quantifiable backup for the feedback from participants during post-study interviews. Many participants said that they would prefer to exercise with a robot partner than a $\mathrm{HP}$. They argued that the robot is not evaluating or judging them while exercising and thus feel more comfortable with a robotic partner than a $\mathrm{HP}$. This participant feedback supports a future application of SAR as a rehabilitation and exercising tool for people with social anxieties, which might prevent them from exercising in groups. Thus robots could facilitate the motivational effects of exercising in groups for such a user population.

\section{Conclusion}

The question of an agent's embodiment is crucial to ask regarding maintenance, cost-benefit ratio, and deployability. Using robots for socially assistive tasks will only be beneficial if they prove to have an advantage compared to other agent representations. Regarding the usage of SAR as exercising partners, we wanted to fill the research gap and provide evidence that a SAR will not only affect the user's perception of the robot but will also enhance the user's motivation to exercise and thus potentially increase the $\mathrm{PA}$. However, the presented evidence needs further approval with long-term interaction studies and unified benchmarks. Therefore, the research community needs to standardize tasks, robots and virtual agents to measure the motivational effects of having SAR

Acknowledgments We would like to thank Feltz et al. for providing their dataset and discussing their work which was supported by grant 1R21HL111916-01A1 from the National Heart, Lung, and Blood Institute 


\section{References}

[1] Wilma A Bainbridge et al. "The benefits of interactions with physically present robots over video-displayed agents". In: International Journal of Social Robotics 3.1 (2011), pp. 41-52.

[2] Christoph Bartneck et al. "Measurement instruments for the anthropomorphism, animacy, likeability, perceived intelligence, and perceived safety of robots". In: International journal of social robotics 1.1 (2009), pp. 71-81.

[3] Adrian E Bauman et al. "Toward a better understanding of the influences on physical activity: the role of determinants, correlates, causal variables, mediators, moderators, and confounders". In: American journal of preventive medicine 23.2 (2002), pp. 5-14.

[4] Nicholas Epley et al. "On seeing human: a three-factor theory of anthropomorphism." In: Psychological review 114.4 (2007), p. 864.

[5] Juan Fasola et al. "A socially assistive robot exercise coach for the elderly". In: Journal of Human-Robot Interaction 2.2 (2013), pp. 3-32.

[6] David Feil-seifer et al. "Defining socially assistive robotics". In: in Proc. IEEE International Conference on Rehabilitation Robotics (ICORR 05. 2005, pp. 465468.

[7] Deborah L Feltz et al. "Cyber buddy is better than no buddy: A test of the Köhler Motivation Effect in exergames". In: GAMES FOR HEALTH: Research, Development, and Clinical Applications 3.2 (2014), pp. 98-105.

[8] Deborah Kendzierski et al. "Physical Activity Enjoyment Scale: Two validation studies." In: Journal of Sport E Exercise Psychology (1991).

[9] James Kennedy et al. "Comparing robot embodiments in a guided discovery learning interaction with children". In: International Journal of Social Robotics 7.2 (2015), pp. 293-308.

[10] Daniel Leyzberg et al. "The physical presence of a robot tutor increases cognitive learning gains". In: Proceedings of the 34th Annual Conference of the Cognitive Science Society. Austin, TX: Cognitive Science Society. 2012.

[11] Jamy Li. "The benefit of being physically present: A survey of experimental works comparing copresent robots, telepresent robots and virtual agents". In: International Journal of Human-Computer Studies 77 (2015), pp. 23-37.

[12] Byron Reeves et al. "The media equation: How people treat computers, television and new media like real people". In: Computers and Mathematics with Applications 5.33 (1997), p. 128.

[13] Astrid M Rosenthal-von der Pütten et al. "Robots or Agents-Neither Helps You More or Less During Second Language Acquisition". In: International Conference on Intelligent Virtual Agents. Springer. 2016, pp. 256-268.

[14] Sebastian Schneider et al. "Exercising with a humanoid companion is more effective than exercising alone". In: Humanoid Robots (Humanoids), 2016 IEEE-RAS 16th International Conference on. IEEE. 2016, pp. 495-501.

[15] Sebastian Schneider et al. "Motivational Effects of Acknowledging Feedback from a Socially Assistive Robot". In: Social Robotics: 8th International Conference, ICSR 2016, Kansas City, MO, USA, November 1-3, 2016 Proceedings. Springer International Publishing, 2016, pp. 870-879.

[16] Stewart G Trost et al. "Correlates of adults' participation in physical activity: review and update". In: Medicine $\mathcal{E}$ Science in Sports E Exercise 34.12 (2002), pp. 1996-2001. 